\title{
HRVFrame: Java-Based Framework for Feature Extraction from Cardiac Rhythm
}

\author{
Alan Jovic ${ }^{1}$ and Nikola Bogunovic ${ }^{1}$ \\ ${ }^{1}$ Department of Electronics, Microelectronics, Computer and Intelligent Systems, \\ Faculty of Electrical Engineering and Computing, University of Zagreb, \\ Unska 3, 10000 Zagreb, Croatia \\ \{alan.jovic, nikola.bogunovic\}@fer.hr
}

\begin{abstract}
Heart rate variability (HRV) analysis can be successfully applied to automatic classification of cardiac rhythm abnormalities. This paper presents a novel Java-based computer framework for feature extraction from cardiac rhythms. The framework, simply called HRVFrame, implements more than 30 HRV linear time domain, frequency domain, time-frequency domain, and nonlinear features. Output of the framework in the form of arff files enables easier medical knowledge discovery via platforms such as RapidMiner or Weka. The scope of the framework facilitates comparison of models for different disorders. The framework can be used for general cardiac disorder analysis as well as for specific cardiac rhythm analysis. Some of the features implemented in the framework can also be applied to other biomedical timeseries. The thorough approach to feature extraction pursued in this work is also encouraged for other types of biomedical time-series.
\end{abstract}

Keywords: heart rate variability; computer framework; nonlinear features; knowledge discovery

\section{Introduction}

Analysis of cardiac abnormalities usually starts with routine diagnostic procedure called electrocardiogram (ECG). The procedure is performed in all types of medical institutions. Detection of anomalies in an ECG can indicate the existence of cardiovascular and other diseases [1]. Heart rate variability (HRV) analysis examines fluctuations in the sequence of cardiac interbeat (RR) intervals. Many types of irregular ECG patterns can be successfully detected by HRV analysis, whereas some patterns (e.g. left and right bundle branch block, nodal rhythm) are known to be practically undetectable due to the similarity with normal rhythm [2].

RR intervals can be obtained from ECG with great accuracy using methods for $\mathrm{R}$ peak detection that are robust to noise [3]. The clinical relevance of HRV analysis was first appreciated in 1965 when it was observed that fetal distress was preceded by alterations in interbeat intervals before any appreciable change in the heart rate itself [4]. HRV analysis using linear time and frequency domain feature was pursued in the 1980-ies and first half of the 1990-ies when it was shown that these features have high descriptive, classification, and predictive capabilities [4]. However, nonstationarities 
and apparent nonlinear behavior of the cardiac rhythm motivated the researchers to explore other approaches for more accurate cardiac rhythm pattern modeling. Timefrequency features and nonlinear features based on chaos theory were applied around the end of the 20th century. Nonlinear methods were designed to fully describe the complex patterns of rhythm disorders [5,6]. In the first decade of the 21 st century, the research turned mostly to the examination of different feature combinations for automatic classification and prediction of cardiac arrhythmias [2,7,8], although a number of new nonlinear features were developed for specific purposes [9].

In cardiac rhythm research there is a problem with the selection of features for optimal arrhythmia description. Namely, the feature space of the heart rhythm is infinite. To reduce the dimensionality to a useful level, researchers base their work either on physiological explicability of the features or on proven mathematical properties of the time-series. Both of these approaches do not guarantee that the entire feature space had been searched. This fact encourages researchers to try to find ever more valuable features and examine their combinations in the analysis of rhythm disorders.

The features can be grouped in several categories depending on the type of the time-series' analysis needed to be performed for their extraction. Most authors agree upon the following categories: linear, time domain; linear, frequency domain; timefrequency domain; nonlinear features [4]. An additional category is also mentioned that analyzes heart rhythm acceleration and deceleration patterns as code words [10]. Some of the good overviews of different features stemming from these categories are provided by authors $[9,11]$.

Linear time and frequency domain features include statistical properties of the heart rhythm (e.g. mean heart rate, standard deviation of the heart rate...), geometrical properties of the RR interval length distribution, and power spectral density estimates in several clinically relevant frequency bands [4]. Time-frequency features do not assume that the time-series is stationary, and are therefore useful in cardiac rhythm analysis. The time-frequency methods mostly employed for arrhythmia detection from ECG and HRV time-series include Short-Time Fourier Transform (STFT), Continuous Wavelet Transform (CWT) and Discrete Wavelet Transform (DWT), with several common types of wavelet functions (e.g. Haar and Morlet wavelet). Out of these three methods, DWT is most often applied to HRV analysis [6].

Nonlinear features have also been applied to HRV analysis. Numerous nonlinear features can be roughly grouped into three categories: chaos and phase space quantification features (e.g. correlation dimension, Lyapunov exponents), entropybased features (e.g. approximate entropy, spectral entropy) and other nonlinear features (e.g. multiscale asymmetry index).

Feature extraction from cardiac rhythm in the works of most authors relies on their own implementation of the features found in literature. However, there are several frameworks and programs known to us at the researchers' disposal that implement some of the features used in the HRV analysis. These include: ECGLab [12], KARDIA [13], BioSig project [14], and HRV Analysis Software [15].

In this work, we develop a novel framework for HRV analysis called HRVFrame that implements most of the known and used features for HRV analysis. It also implements most of the recently explored nonlinear complexity measures. The framework is intended to act as a bridge between cardiac rhythm data records and 
medical knowledge discovery using statistical analysis and machine learning methods. The strong point of the framework is the number of implemented features, which allows researchers to easily compare the results with other authors. Our framework is still a work in progress, albeit at an advanced stage. Previous versions of the framework have allowed us to analyze with high accuracy several types of cardiac disorders $[16,17]$.

The paper is organized as follows. In section 2, we provide an overview of the framework. A comparison of our framework with known tools and programs mentioned earlier is given in section 3. In section 4 we discuss possible applications of the framework. Section 5 concludes the paper.

\section{Framework Overview}

HRVFrame is an extensive Java-based framework containing many features covered in the HRV analysis literature. Its main purpose is to extract features from cardiac rhythm records and store them as feature vectors prepared for further knowledge discovery. At present, the process of feature extraction is performed offline and the framework has not been integrated in any particular knowledge-based platform. The whole process of cardiac rhythm records analysis using HRVFrame is shown in Fig. 1.



Fig. 1. Cardiac rhythm analysis using the HRVFrame computer framework

HRVFrame is organized into three major logical parts: input, feature calculation, and output.

\subsection{Data Input}

The input part of the framework is designed to take cardiac rhythm records, to allow the selection of features and specification of features' parameters. Cardiac rhythm records in the form of textual ASCII files are acceptable as input files. The input file structure is the same as the one provided by the PhysioNet tool rdann [18], 
which is a part of the WFDB Software Package. The files should contain the information on the times of R peaks, types of beats, and optional rhythm annotations. All beat and rhythm annotations used in PhysioBank databases are supported by the framework. Other information such as sample number and database-specific comments are not supported because they are considered irrelevant for HRV analysis. Also acceptable is the input format where cardiac beat types and/or rhythm annotations are unknown.

Some features have parameters that determine the calculation procedure. Currently, the framework accepts a list of desired features as executable program parameters. Visual interface for feature and parameter selection is planned in the future. HRVFrame also accepts the list of cardiac rhythm files for which the features should be calculated. In addition, some other extraction process parameters can be specified, e.g. start time or starting interval, end time or ending interval.

\subsection{Feature Calculation}

HRVFrame is used to calculate values for the specified HRV features. The list of currently supported features along with the references that provide theory for their calculation is provided in Table 1. Linear HRV features have been implemented according to the European Society of Cardiology Guidelines for HRV [4]. Because the guidelines from 1996 do not deal in detail with newly developed nonlinear features, a thorough search of the literature was performed. Most of the nonlinear features applied in HRV research were implemented in the framework.

Table 1. Features implemented in the HRVFrame framework.

\begin{tabular}{lll}
\hline Feature(s) & Category & Parameters \\
\hline $\begin{array}{l}\text { mean,SDNN, RMSSD, SDANN, } \\
\text { Fano factor, Allan factor [2,4] }\end{array}$ & $\begin{array}{l}\text { Linear, time } \\
\text { domain, statistical }\end{array}$ & $\begin{array}{l}\text { SDANN: number of segments; } \\
\text { pNNX: miliseconds } X \text {; Fano } \\
\text { factor, Allan factor: counting } \\
\text { time }\end{array}$ \\
\hline HRV triangular index, TINN [4] & $\begin{array}{l}\text { Linear, time } \\
\text { domain,geometric }\end{array}$ & \\
\hline $\begin{array}{l}\text { Total PSD, ULF, VLF, LF, HF, } \\
\text { LF/HF [4] }\end{array}$ & $\begin{array}{l}\text { Linear, frequency } \\
\text { domain }\end{array}$ & $\begin{array}{l}\text { Fast Fourier Transform (FFT); } \\
\text { window (Hanning, Hamming, } \\
\text { none); Burg AR model; model } \\
\text { order; frequency band selection }\end{array}$ \\
\hline $\begin{array}{l}\text { Haar wavelet standard deviation } \\
\text { [6] }\end{array}$ & $\begin{array}{l}\text { Time-frequency } \\
\text { domain, DWT }\end{array}$ & \begin{tabular}{l} 
Scale \\
\hline Largest Lyapunov exponent [19]
\end{tabular} \\
$\begin{array}{l}\text { Nonlinear, phase } \\
\text { space }\end{array}$ & $\begin{array}{l}\text { Embedding dimension, } \\
\text { trajectory length }\end{array}$ \\
\hline Correlation dimension $D_{2}[20]$ & $\begin{array}{l}\text { Nonlinear, phase } \\
\text { space }\end{array}$ & Embedding dimension, lag \\
\hline Spatial filling index [21] & $\begin{array}{l}\text { Nonlinear, phase } \\
\text { space }\end{array}$ & Embedding dimension, lag \\
\hline
\end{tabular}




\begin{tabular}{lll}
\hline Central tendency measure [22] & $\begin{array}{l}\text { Nonlinear, phase } \\
\text { space }\end{array}$ & Embedding dimension, lag \\
\hline $\begin{array}{l}\text { Standard deviations ratio } \\
\text { (SD1/SD2), CSI, CVI [23,24] }\end{array}$ & $\begin{array}{l}\text { Nonlinear, phase } \\
\text { space }\end{array}$ & \\
\hline Sequential trend analysis [12] & $\begin{array}{l}\text { Nonlinear, phase } \\
\text { space }\end{array}$ & \\
\hline $\begin{array}{l}\text { Detrended fluctuation } \\
\text { analysis (DFA) } \alpha_{1}, \alpha_{2}[25]\end{array}$ & $\begin{array}{l}\text { Nonlinear, fractal } \\
\text { estimates }\end{array}$ & \\
\hline Hurst exponent [6] & $\begin{array}{l}\text { Nonlinear, fractal } \\
\text { estimates }\end{array}$ & \\
\hline Higuchi's fractal dimension [11] & $\begin{array}{l}\text { Nonlinear, fractal } \\
\text { estimates }\end{array}$ & kmax \\
\hline Approximate entropy (ApEn) [26] & Nonlinear, entropy & $\begin{array}{l}\text { Embedding dimension, radius, } \\
\text { maximum (Y/N) }\end{array}$ \\
\hline Sample entropy (SampEn) [27] & Nonlinear, entropy & $\begin{array}{l}\text { Embedding dimension, radius, } \\
\text { maximum (Y/N) }\end{array}$ \\
\hline Multiscale sample entropy [28] & Nonlinear, entropy & $\begin{array}{l}\text { Scale, embedding dimension, } \\
\text { radius, maximum (Y/N) }\end{array}$ \\
\hline Rényi entropy [21] & Nonlinear, entropy & Order \\
\hline Spectral entropy [8] & Nonlinear, entropy & Frequency band selection \\
\hline Multiscale asymmetry index [29] & Nonlinear, other & Scale \\
\hline
\end{tabular}

Features are calculated for one segment of RR intervals at a time. The length of the segment can be given in the number of RR intervals, or in the number of seconds. In addition, framework also supports the analysis of the whole record as a single segment. This is particularly useful for the features that require a larger number of intervals for correct calculation, such as fractal dimension estimates.

\subsection{Framework Output}

The framework allows users to first create an .arff file that only contains the declaration of features (attributes in .arff file format). After the feature calculation process for an RR segment is complete, the framework creates output feature vectors and stores them in the .arff file. Data are appended to the end of the file as long as the new segments and records are continued to be analyzed. The arff file can then be used by several data mining and knowledge discovery platforms like Weka [30] and RapidMiner [31] for disorder classification.

\section{Comparison to Similar Work}

The implementation of HRV toolkit named ECGLab by authors [12] allows biomedical engineers to explore and analyze cardiac rhythm in Matlab environment. The first version of the software contained routines for $\mathrm{R}$ peak detection out of the 
ECG record as well as for loading of already prepared ASCII files that contain RR intervals. The implemented linear time domain features include mean, SDNN, RMSSD, and pNN50. Linear frequency domain analysis was also implemented that includes FFT and the use of five types of window functions, AR model and LombScargle periodogram. Nonlinear measures include the analysis of Poincaré plot and sequential trend analysis. In later works, the same group of authors implemented additional features. These include time-frequency analysis using Short-Time Fourier Transform (STFT), CWT scalogram, and time-variant AR modeling [32] as well as a detailed tool for DFA [33].

The advantage of the ECGLab software is its integration with Matlab, which offers numerous possibilities for data manipulation and visualization. Implementation is also very fast. The disadvantages include dependance of the toolkit on Matlab availability and its classification capabilities and relatively small number of features compared to our work. Matlab is primarily a platform for engineering and signal analysis. It is not specialized for knowledge discovery such as other platforms, it is not freely available and is a relatively closed system.

KARDIA system is a recently developed Matlab software for cardiac interbeat interval analysis [13]. It has visual interface and enables analysis of several rhythm records at a time. The entire code of the software is placed in a Matlab $m$-file. KARDIA allows standard linear time and frequency analysis of cardiac records. Frequency domain analysis includes the estimation of power spectral density using either FFT with window function or AR parametric model with Burg algorithm. The software can also analyze phasic cardiac responses (PCR) using fractional cycle count theory, as explained in [34]. In addition, DFA can be performed to estimate fractal scaling properties of the rhythm. When compared to our framework, KARDIA allows extraction of fewer features and, like ECGLab, has similar advantages and disadvantages because of its implementation in Matlab.

Project BioSig is an interesting initiative that aims to standardize biomedical signal processing tools and allow easier file format conversions, results reproducibility, and comparison [14]. The current implementation is open source, programmed in $\mathrm{C} / \mathrm{C}++$. Although some parts are stand-alone, other tools work only with the Matlab platform, which the authors recognize as a disadvantage. The primary application field of the software is in brain-computer interaction (BCI), including research of brain's coupling properties using EEG data. It also allows linear time and frequency domain HRV analysis. The authors point out that more advanced ECG and HRV analysis are dependent on experts, which means that the software should be upgraded for that purpose. The main advantage of BioSig is that it is open source and upgradable.

HRV Analysis Software [15] is freely-available Windows software for HRV analysis with visual interface. Originally, the software components were written in Matlab. The source files were later recompliled in $\mathrm{C}$ and the software is now standalone. Features extracted by the software include linear time domain (mean, SDNN, RMSSD, pNN50), linear frequency domain nonparametric FFT and parametric AR spectral estimates, and nonlinear Poincaré plot standard deviations SD1 and SD2. The software enables RR interval data input from ASCII files, data output to commaseparated values file and also a printable report sheet. Data input in the form of RR intervals' lengths can be loaded from ASCII file. This format does not support supervised learning and classification, only statistical analysis of the obtained results. 
Also, the lack of other time-series analysis features used in HRV research does not support scientific exploration and comparison of feature combinations.

To summarize, most tools have only limited scientific value in HRV analysis because of the insufficient number of implemented features. The use of proprietary software (Matlab) limits the use of ECGLab, KARDIA, and BioSig. For future research and development, BioSig seems like a promising solution. However, at this stage of development, it is still not particularly useful in advanced HRV analysis.

\section{Framework Applications}

\subsection{Scientific Applications}

The main application of the framework is in preparation of cardiac rhythm data for automated classification tasks. HRVFrame is oriented toward scientific exploration of different feature combinations for optimal models of cardiac abnormalities. It can be used for result comparison from different authors and for investigation of the research results reported by the authors in this domain. It should be however noted that this type of analysis requires that the protocol was clearly stated by the authors and that the used data is publicly available, which is often not a case.

The framework is of primary use to the scientific community, including medical researchers and computer scientists. Medical researchers are able to compare different models of cardiac abnormalities based on rhythm features and use the ones that are sufficiently accurate and with clear interpretation. Computer scientists have the opportunity to explore the obtained feature vectors and utilize different machine learning algorithms for the analysis. Feature selection methods and classification algorithms are used in order to obtain accurate and interpretable models.

\subsection{Analysis of Cardiac Disorders and Arrhythmic Patterns}

The framework can be used for two types of HRV analysis. The first one is the analysis of cardiac disorders, in which one specifies which disorder is represented by the cardiac rhythm records given to the framework. The framework then assumes that the disorder is present in the whole record, which is usually true. The disorder type is considered as the target feature, having any user-selected labels for different analyzed disorders. Any type of disorder can be studied based on the rhythm (e.g. congestive heart failure, cardiac arrhythmia).

The second type of analysis one can pursue is the analysis of the exact rhythm present in the records, analyzed segment by segment. Each segment, depending on its length, can contain a number of different rhythm annotations. Sometimes a segment that is annotated as normal rhythm contains anomalous beats (e.g. premature ventricular contractions). This segment is not considered to have normal rhythm and is labeled as abnormal depending on the nature of the anomalous beats.

The framework always designates one type of rhythm per segment. When several different rhythms or anomalous beats are encountered, the framework selects the 
assignment based on rhythm priority. The priorities can be defined in a separate file by the user. Default priority for currently supported abnormalities is shown in Table 2 in descending order. The priorities of the rhythm level always take precedence over the priorities of the beat level. All abnormalities have higher priority than normal rhythm. At the moment, sinus tachycardia and sinus arrhythmia are considered to be normal rhythms. Ventricular fibrillation (VF) is currently not considered due to great difficulty in finding significant ventricular responses during VF episodes.

Table 2. Default priority for supported cardiac rhythm and beat abnormalities.

\begin{tabular}{cl}
\hline & Ventricular flutter $->$ ventricular tachycardia $->$ ventricular bigeminy $->$ \\
Rhythm & ventricular trigeminy $->$ Mobitz II second degree heart block $->$ idioventricular \\
level & rhythm $->$ atrial flutter $->$ atrial fibrillation $->$ supraventricular tachyarrhythmia $->$ \\
& AV nodal rhythm $->$ atrial bigeminy $->$ atrial trigeminy $->$ paced rhythm $->$ sinus \\
& pause $->$ sinus bradycardia \\
\hline Beat & Compensated couplet $->$ decompensated couplet $->$ PVC $->$ fusion $->$ ventricular \\
level & ectopy $->$ PVC and PAC $->$ PAC $->$ LBBB $->$ RBBB $->$ normal \\
\hline
\end{tabular}

\subsection{Application in Other Domains}

HRVFrame contains many features used by researchers in HRV analysis. It is interesting to note that the majority of the features are not specific for cardiac rhythm analysis and can be used in the analysis of other biomedical time-series (ECG, EEG, EMG, gait, skin resistance...). Therefore, constructing frameworks for the analysis of other biomedical time-series is not so difficult with the experience acquired in the construction of this framework. A careful consideration of official medical guidelines for the domain of interest and several important domain-specific papers that deal with feature extraction is a good start for implementation of the framework for other biomedical rhythms.

Application of our framework in non-cardiac domain is also possible if one researches couplings between several different biomedical time-series. Frameworks for other domain would in this case be very helpful for obtaining as much information as possible for better explanation of the researched disorders.

\subsection{Availability}

The current version of the framework is available free-of-charge, and only for noncommercial, scientific purposes. The framework is not open source at the moment. Because the code is written entirely in Java, the framework can be used on any operating system that supports Java, version 1.6 or higher. Please contact the author by e-mail: alan.jovic@fer.hr for further instructions. 


\section{Conclusion}

A novel framework for HRV analysis is presented in this work. The framework allows researchers to thoroughly analyze cardiac rhythm records with different features and their combinations. It can be used in the analysis of cardiac disorders, arrhythmias, and in other biomedical domains. The main advantage of the framework compared to the existing solutions lies in the larger number of implemented features. Future work will be focused on improving the interface of the framework and on implementation of other existing nonlinear methods for cardiac rhythm analysis.

\section{References}

1. Garcia, T.B., Holtz, N.E.: 12-Lead ECG: The Art of Interpretation. Jones and Bartlett Publishers, Sudbury, MA, USA (2001)

2. Tsipouras, M.G., Fotiadis, D.I.: Automatic arrhythmia detection based on time and timefrequency analysis of heart rate variability. Comp. Meth. Prog. Biomed. 74(2), 95--108 (2004)

3. Adnane, M., Jiang, Z., Choi, S.: Development of QRS detection algorithm designed for wearable cardiorespiratory system. Comp. Meth. Prog. Biomed. 93, 20--31 (2009)

4. Malik, M., Bigger, J.T., Camm, A.J., Kleiger, R.E., Malliani, A., Moss, A.J., Schwartz, P.J.: Heart rate variability guidelines: Standards of measurement, physiological interpretation, and clinical use. Eur. Heart J. 17(3), 354--381 (1996)

5. Ivanov, P.C., Amaral, L.A., Goldberger, A.L., Havlin, S., Rosenblum, M.G., Struzik, Z.R., Stanley, H.E.: Multifractality in human heartbeat dynamics. Nature 399, 461--465 (1999)

6. Teich, M.C., Lowen, S.B., Jost, B.M., Vibe-Rheymer, K., Heneghan, C.: Heart-Rate Variability: Measures and Models. In: Akay, M. (ed.) Nonlinear Biomedical Signal Processing, vol. II, Dynamic Analysis and Modeling, ch. 6, pp. 159--213. IEEE Press, New York (2001)

7. Kudaiberdieva, G., Görenek, B., Timuralp, B.: Heart rate variability as a predictor of sudden cardiac death. The Anatolian journal of cardiology 7, 68--70 (2007)

8. Asl, B.M., Setarehdan, S.K., Mohebbi, M.: Support vector machine-based arrhythmia classification using reduced features of heart rate variability signal, Artif. Intell. Med. 44(1), 51--64 (2008)

9. Xia, H., Zhao, X., Bains, J., Wortham, D.C.: A Review of Diagnosis Methods for Heart Rhythm Disorders. In: Evans, B.M. (ed.) Biomedical Science \& Engineering Conference BSEC 2009, pp. 1--4. IEEE Press, New York (2009)

10. Yang, A.C.-C., Hseu, S.-S., Yien, H.-W., Goldberger, A.L., Peng, C.-K.: Linguistic Analysis of the Human Heartbeat Using Frequency and Rank Order Statistics. Phys. Rev. Lett. 90(10), 108103 (2003)

11. Acharya, R.U., Joseph, K.P., Kannathal, N., Lim, C.M., Suri, J.S.: Heart rate variability: a review. Med. Bio. Eng. Comput. 44, 1031--1051 (2006)

12. de Carvalho, J.L.A., da Rocha, A.F., Nascimento, F.A.O., Neto, J.S., Junqueira, L.F.: Development of Matlab Software for Analysis of Heart Rate Variability. In: Proc 6th Int Conf Sig Proc ICSP 2002, pp. 1488--1491, IEEE Press, Beijing, China (2002)

13. Perakakis, P., Joffily, M., Taylor, M., Guerra, P., Vila, J.: KARDIA: A Matlab software for the analysis of cardiac interbeat intervals. Comp. Meth. Prog. Biomed. 98, 83--89 (2010)

14. Schlögl, A., Vidaurre, C., Hofer, E., Wiener, T., Brunner, C., Scherer, R., Chiarugi, F.: Biosig - standardization and quality control in biomedical signal processing using the 
biosig project. In: Encarnação, P., Veloso, A. (eds.) Proc. 1st Int. Joint Conf. Biomedical Engineering Systems and Technologies BIOSTEC 2008, pp. 403--409, IEEE Press, Funchal, Madeira, Portugal (2008)

15. Niskanen, J.-P., Tarvainen, M.P., Ranta-aho, P.O., Karjalainen, P.A.: Software for advanced HRV analysis. Comp. Meth. Prog. Biomed. 76, 73--81 (2004)

16. Jovic, A., Bogunovic, N.: Random Forest-Based Classification of Heart Rate Variability Signals by Using Combinations of Linear and Nonlinear Features. In: Bamidis, P.D., Pallikarakis, N. (eds.) Proc. XII Mediterranean Conf. Medical and Biological Engineering and Computing MEDICON 2010, pp. 29--32, Springer, Berlin (2010)

17. Jovic, A., Bogunovic, N.: Electrocardiogram analysis using a combination of statistical, geometric, and nonlinear heart rate variability features. Artif. Intell. Med., in press, doi:10.1016/j.artmed.2010.09.005

18. PhysioNet tool $r$ dann, http://www.physionet.org/tutorials/physiobank-text.shtml\#ann

19. Rosenstien, M., Colins, J.J., de Luca, C.J.: A practical method for calculating largest Lyapunov exponents from small data sets. Physica D 65, 117--134 (1993)

20. Grassberger P., Procaccia, I.: Measuring the strangeness of strange attractors. Physica D: Nonlinear Phenomena 9(1-2), 189--208 (1983)

21. Faust, O., Acharya, R.U., Krishnan, S.M., Min L.C.: Analysis of cardiac signals using spatial filling index and time-frequency domain. BioMedical Engineering OnLine 3, 30 (2004)

22. Seker, R., Saliu, S., Birand, A., Kudaiberdieva, G.: Validity Test for a Set of Nonlinear Measures for Short Data Length with Reference to Short-Term Heart Rate Variability Signal. Journal of Systems Integration 10, 41--53 (2000)

23. Toichi, M., Sugiura, T., Murai, T., Sengoku, A.: A new method of assessing cardiac autonomic function and its comparison with spectral analysis and coefficient of variation of R-R interval. J Auton Nerv Syst 62(1-2), 79--84 (1997)

24. Lin, C.-W., Wang, J.-S., Chung, P.-C.: Mining Physiological Conditions from Heart Rate Variability Analysis. IEEE Comput. Intell. Mag. 5(1), 50--58 (2010)

25. Peng, C.-K., Havlin, S., Stanley, H.E., Goldberger, A.L.: Quantification of scaling exponents and crossover phenomena in nonstationary heartbeat time series. Chaos 5(1), 82--87 (1995)

26. Pincus, S.M., Goldberger, A.L.: Physiological time series analysis: what does regularity quantify? Am J Physiol 266, 1643--1656 (1994)

27. Richman, J.S., Moorman, J.R.: Physiological time-series analysis using approximate entropy and sample entropy. Am J Physiol Heart Circ Physiol 278, 2039--2049 (2000)

28. Costa, M., Goldberger, A.L., Peng, C.-K.: Multiscale entropy analysis of biological signals. Phys. Rev. E 71, 021906 (2005)

29. Costa, M., Goldberger, A.L., Peng, C.-K.: Broken asymmetry of the human heartbeat: Loss of time irreversibility in aging and disease. Phys. Rev. Lett. 95, 198102 (2005)

30. Witten I.H., Frank, E.: Data mining: Practical machine learning tools and techniques. Morgan Kaufmann, San Francisco (2005)

31. RapidMiner, http://rapid-i.com/content/view/181/190/

32. de Carvalho, J.L.A., da Rocha, A.F., Junqueira, L.F., Neto, J.S., Santos, I., Nascimento, F.A.O.: A tool for time-frequency analysis of heart rate variability. In: Proc. 25th Annual Int. Conf. IEEE EMBS 2003, pp. 2574--2577, IEEE Press, New York (2003)

33. Leite, F.S., da Rocha, A.F., Carvalho, J.L.A.: Matlab software for detrended fluctuation analysis of heart rate variability. In: Proc. 3rd Int. Conf. Bio-inspired Systems and Signal Processing BIOSIGNALS 2010, pp. 225--229, INSTICC, Valencia (2010)

34. Dinh, T., Perrault, H., Calabrese, P., Eberhard, A., Benchetrit, G.: New statistical method for detection and quantification of respiratory sinus arrhythmia. IEEE Trans. Biomed. Eng. 46, 1161--1165 (1999) 